\title{
Feature extraction-based prediction of tool wear of Inconel 718 in face turning
}

\author{
M Murua, A Suárez, L N López de Lacalle, R Santana and A Wretland
}

\begin{abstract}
Tool wear is a recurring topic in the cutting field, so obtaining knowledge about the tool wear process and the capability of predicting tool wear is of special importance. Cutting processes can be optimised with predictive models that are able to forecast tool wear with a suitable level of accuracy. This research focuses on the application of some regression approaches, based on machine learning techniques, to a face-turning process for Inconel 718. To begin with, feature extraction of the cutting forces is considered, to generate regression models. Subsequently, the regression models are improved with a reduced set of features obtained by computing the feature importance. The results provide evidence that the gradient-boosting regressor allows an increment in the wear prediction accuracy and the random forest regressor has the capability of detecting relevant features that characterise the turning process. They also reveal higher accuracy in predicting tool wear under high-pressure cooling as opposed to conventional lubrication.
\end{abstract}

Keywords: tool wear prediction, machine learning, face turning of superalloys.

\section{Introduction}

The wear of machining tools in the process of turning has been extensively studied in recent decades as it is a relevant factor that affects machined surface characteristics. During the machining process, the surface can become destroyed and this can be decisive for the properties of the manufactured parts. In view of this, surface integrity has come to be considered the most important parameter for evaluating the quality of a machined $\operatorname{part}^{[1]}$. Moreover, tool wear is a major problem in the machining of nickel-based superalloys due to the high temperature and stresses generated in the tool-chip interface ${ }^{[8]}$. As a consequence, knowledge of the tool wear system and the capability to forecast tool wear are of great importance.

Tool wear prediction has attracted the interest of scholars as it is considered important in industry for higher productivity and product quality. Not only are the quality and integrity of the machined parts essential, but minimising material waste is also crucial for sustainable manufacturing. In an investigation into analytical models for tool wear prediction in turning, Attanasio et al ${ }^{[3]}$ proposed the use of their model in production management software to replace the tool during production. Similarly, a tool wear predictive model was employed $\mathrm{in}^{[11]}$ to monitor tool wear in the milling process. This made it possible to prevent degradation in the machining process. Therefore, accurate predictive models of tool wear are needed for use in online tool condition monitoring systems ${ }^{[4]}$.

Inconel 718 is a nickel-iron-based superalloy that is mainly applied in the hot section of turbine machinery and nuclear reactors. These materials are classified as extremely difficult to cut as, during the machining process, the interaction between the tool and the workpiece causes severe deformation in the workpiece ${ }^{[34]}$. Therefore, tool wear and tool life are key factors when machining these kinds of alloys ${ }^{[18]}$. They also present exceptional properties, such as high strength and corrosion resistance at high temperatures, as well as good creep behaviour ${ }^{[29]}$. This research focuses on predicting tool wear based on some regression approaches using features extracted from the cutting forces. Although the prediction of a phenomenon is a preliminary step towards optimising a process, this investigation also shows how different characteristics of the material influence the amount of wear in the different passes of the face turning. In addition, a novel feature extraction method is introduced that identifies peaks in the time series, describing the cutting forces, and computes descriptive statistics from these peaks.

Different types of wear are studied in the literature, such as flank wear and notch wear. The flank face is the area of the tool over which the surface produced on the workpiece passes and this surface is where flank wear occurs ${ }^{[2]}$. Flank wear is the most common type of wear and appears because of abrasion caused by hard constituents in the workpiece material. It starts at the cutting tip and then widens as the contact area increases, hence forming the wear land. The width, shape and growth of the wear land depends on the tool material, the workpiece material and the cutting parameters ${ }^{[26]}$. Notch wear is a common wear phenomenon in the machining of heat resistant superalloys (HRSAs). It occurs due to adhesion (pressure welding of chips) and a deformation-hardened surface. The notch wear appears outside of the cutting depth ${ }^{[19]}$. Although the effect of notch wear is considered important, this investigation focuses on predicting the flank wear of the tool. The prediction of this phenomenon has some associated difficulties due to the large number of parameters involved in the process. It should be added that available experimental wear data are noisy and a data preprocessing step is necessary.

In an investigation carried out with Inconel 718 to determine the effect of grain size and hardness on the wear of the machining tool, Olovsjö et $a l^{[19]}$ arrived at the conclusion that, for materials with

\section{Submitted 30.11.17 / Accepted 25.04.18}

Maialen Murua and Alfredo Suárez are with the Advanced Manufacturing Department, Tecnalia R\&l, Paseo Mikeletegi 7, 20009 San Sebastián, Spain.

Luis Norberto López de Lacalle is with the Faculty of Engineering of Bilbao, Department of Mechanical Engineering, University of the Basque Country, 48013 Bilbao, Spain.

Roberto Santana is with the Intelligent Systems Group, Department of Computer Science and Artificial Intelligence, University of the Basque Country, 20018 San Sebastián, Spain.

Anders Wretland is with GKN Aerospace Engine Systems $A B, 46181$ Trollhättan, Sweden. 
small grains, notch wear was less prevalent than for two materials with large grains. The authors also concluded that hardness was not related to flank wear and that a modest influence could be associated with the grain size. However, the general result was that the hardness associated with a smaller grain size did not increase wear ${ }^{[18]}$. Nevertheless, the aforementioned investigations ignored any mathematical framework and focused on comparing tool wear measurements between different states.

The wear of the machining tool in the process of turning has been the subject of a vast number of studies and investigation $s^{[7,26,19]}$. These studies have ranged from the prediction of flank wear, using the force ratio, to a mathematical model that established a relationship between tool wear land area and component forces. The investigations were based on mathematical modelling and they did not make use of artificial intelligence tools. However, for modelling different phenomena relevant to the machining process, some scholars have proposed the use of soft computing techniques ${ }^{[17,9]}$. Some significant characterisations of the machining process can be found in the literature that are based on response surface methodology (RSM), artificial neural networks (ANNs), Bayesian networks (BNs) and multiple regression $(\mathrm{MR})^{[34]}$

Another common approach for predicting tool wear is the support vector machine (SVM) method ${ }^{[25,27]}$. Shi and Gindy ${ }^{[25]}$ developed a new tool wear predictive model through a combination of least squares support vector machines (LS-SVMs) and the principal component analysis (PCA) technique. PCA was first proposed to extract features from multiple sensory signals acquired from the machining process. Finally, an LS-SVM-based tool wear prediction model was constructed by learning the correlation between extracted features and the actual tool wear. There are also recent contributions to the prediction of tool wear that use wear rate models ${ }^{[16,31]}$. In an investigation carried out to estimate the wear of ceramic and coated carbide tools in the turning of Inconel 625, Usui's wear rate model was used. This model is based on the equation of adhesive wear and involves several factors, such as temperature, stress and sliding velocity ${ }^{[33]}$. Feed rate, cutting speed and depth of cut are considered as input parameters.

More closely related to this investigation, Kilundu et a ${ }^{[13]}$ explored the use of data mining techniques for tool condition monitoring and metal cutting. The tool condition was classified using five classes, where 22 different signals were monitored throughout the experiment. Four classification techniques were employed in the dataset: decision trees, Bayesian networks, $k$-nearest neighbours and neural networks. The validation of the approach was performed by means of a confusion matrix. Similarly, decision trees were used in ${ }^{[21]}$ to predict the roughness in face milling. The goal of the aforementioned study was to offer engineers a decision-making system to choose the best cutting tool in milling. Nonetheless, this kind of approach has not yet been used in relation to the specific problem of predicting tool wear.

The remainder of this paper is organised as follows: Section 2 introduces the experimental set-up of the investigation carried out. Section 3 introduces the proposed approach to wear prediction, based on the novel features that characterise the face-turning process and the regression methods applied. Section 4 explains the validation of the proposal and analyses and discusses the results. Finally, Section 6 presents the concluding remarks.

\section{Experimental set-up}

Inconel 718 is a nickel-iron-based superalloy that in this experimental procedure contained $53.6 \%$ nickel and $18.3 \%$ chromium. A slice cut from an original superalloy bar, $126 \mathrm{~mm}$ in diameter and $75 \mathrm{~mm}$ in width, was employed, with a $21 \mathrm{~mm}$ centre hole that was introduced to facilitate the turning tests. Heat treatment, heating and cooling, were applied for the specific purpose of intentionally altering the grain size and hardness. This process included techniques such as annealing and precipitation hardening and was applied to the material, Inconel 718, to obtain two different microstructures that differed in grain size (Table 1): large grain aged (LGA) and small grain aged (SGA).

\section{Table 1. Grain size and microhardness of Inconel 718}

\begin{tabular}{|c|c|c|}
\hline & LGA & SGA \\
\hline Grain size $(\mu \mathrm{m})$ & 130 & $15-32$ \\
\hline Microhardness $(\mathrm{HV})$ & 493 & 497 \\
\hline
\end{tabular}

The face-turning operation was developed using a four-axis CMZ TC25BTY turning centre. Each test had the same material removed, with a total spiral cutting length (SCL) of $727 \mathrm{~m}$, divided into six passes. After each of the passes, the process of turning was stopped and the wear of the tool $(\mu \mathrm{m})$ was measured. The machining tool employed was an uncoated standard cemented carbide tool (Figure 1(a)). The tool wear measurement procedure was developed as follows: each of the pictures of the six passes were taken using the two cameras installed; files were created from all of the pictures using AutoCAD software; and picture dimensioning was performed where the flank wear was measured at nine points, while the notch wear was measured at the maximum value. From the nine points, the average and the maximum wear were stored as variables. Figure 1(b) shows an image of the tool used in the experiment where wear measurements were made at different points.

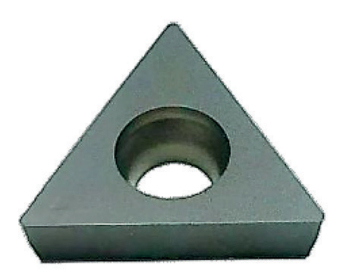

(a)

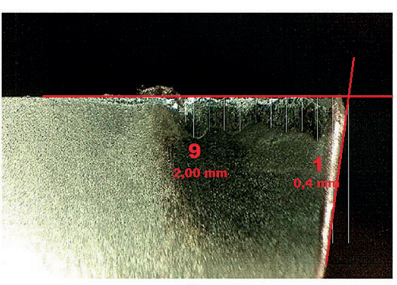

(b)
Figure 1. The tool employed in the experimental procedure: (a) cemented carbide tool employed; and (b) flank wear measurements

The monitoring of the forces was carried out using a Kistler 9129A dynamometer. The total force induced by the action of the cutting tool on the workpiece was called $F$ (see Figure 2). The resulting cutting force, $F$, was broken down into three components: the cutting force $F_{y}$ was the component of the total force $F$ in the direction of the cutting speed, which was tangential; $F_{z}$ was in the orthogonal direction to the cutting speed and did not consume power (passive force); and the component $F_{x}$ was in the radial direction ${ }^{[30]}$.

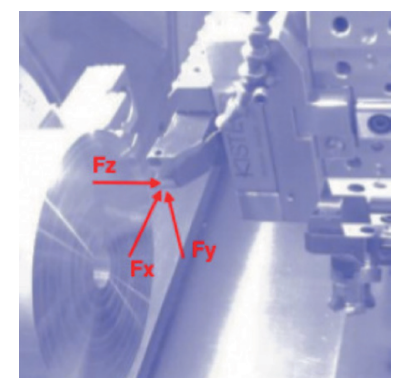

Figure 2. Cutting forces $F_{x}, F_{y}$ and $F_{z}$ in the face-turning operation applied to Inconel 718 
The cutting parameters of the face-turning operation used in all of the experiments considered were the same: the entering angle $\left(91^{\circ}\right)$, the rake angle $\left(0^{\circ}\right)$, the inclination angle $\left(0^{\circ}\right)$, the nose radius $(0.4 \mathrm{~mm})$, the cutting speed $(30 \mathrm{~m} / \mathrm{min})$, the feed rate $(0.1 \mathrm{~mm} / \mathrm{rev})$ and the cutting depth $(2 \mathrm{~mm})$.

The turning process involves the generation of high cutting forces and temperatures and lubrication becomes critical in order to minimise the effects of these forces and temperatures on the cutting tool and workpiece. In this case, conventional and high-pressure cooling (HPC) were used. HPC has attracted interest in industry and it has been shown that high pressure between 60 and 200 bars is a feasible pressure range in terms of both cost and benefit ${ }^{[28]}$.

The analysis was based on data collected from experiments in which different types of material and experimental conditions were used. More specifically, these were the Inconel 718 LGA and SGA states, the forces $F_{x}, F_{y}$ and $F_{z}$ and the maximum flank wear.

After an initial exploration of the data, it was found that a preprocessing step was needed to remove noisy data that was not relevant to the analysis. In the experimental trials, after each of the passes, the process of turning was stopped. As a result, at the beginning and end of each pass, the force increased until it achieved a specific range of values and then decreased significantly. Since these force values were produced by the transition between passes and not by the characteristics of the wear process, an interval of values was set that defined the data that would be considered as relevant and those points that would be removed. Equation (1) describes the threshold of the values that were considered feasible:

$$
\text { thr }=\mu-k \sigma
$$

Here, $\mu$ is the mean of the forces, $\sigma$ is the standard deviation and $k$ is a positive constant value $(k=3)$. Force values below the threshold described by Equation (1) were removed.

\section{Description of the proposed approach}

The presented problem of predicting the flank wear was addressed as a regression task, where the regression was based on a number of features that describe the face-turning process. The proposed machine learning-based approach consisted of first extracting a number of novel features that characterised the face-turning process and then applying different regressors to predict the flank wear, using the extracted features as input variables. Afterwards, feature selection was carried out by employing the feature importance to select a subset of the original features. The proposed machine learning approach was carried out in the following stages: initial feature extraction; application of different machine learning approaches to the regression problem; feature selection by computation of future importance; and application of the regressors to the reduced set of features.

\subsection{Feature extraction}

In machine learning, the algorithms construct associations between several input parameters called features and an output variable of interest ${ }^{[15]}$. A feature extraction procedure builds new variables from the original set of input variables in such a manner that they are relevant and non-redundant ${ }^{[5]}$. That is, given a feature space $x_{i} \in \mathbb{R}^{N}$, the aim is to find a mapping from $\mathbb{R}^{N}$ to $\mathbb{R}^{M}$ with $M<N$, such that the transformed feature vector preserves the information in $\mathbb{R}^{N}$. Another common dimensionality reduction technique is the feature selection approach. Given a feature set $\mathbf{x}=\left\{x_{i} \mid i=1, \ldots, N\right\}$, the aim is to find a subset $\mathbf{x}_{\mathrm{M}}=\left\{x_{1 p}, x_{i 2}, \ldots, x_{i M}\right\}$ with $M<N$ that minimises the error in regression/classification tasks.
The input data of the models, the force signals, were time series in which each observation contained a series of 500,000 data points, listed in time order. It was essential to reduce the dimensionality of the data, but it was difficult to know in advance the data points that were relevant to predict the flank wear so as to select a subset of the features. Thus, a feature extraction technique was applied, transforming the data into a lower-dimensional space. However, feature selection was applied by computing the feature importance after the prediction models were built. The aim at this stage was to improve the quality of the predictive models by keeping the most informative features. The methodology carried out to extract the features is explained below.

The identification and analysis of peaks in a given time series is relevant in many applications ${ }^{[23]}$. Peaks indicate significant events, such as a sudden decrease or increase and sharp rises. A data point in a time series is a local peak if it is a large local maximum (or minimum) within a window. A point is considered a maximum peak if it is preceded (to the left) and followed (to the right) by a lower value. A point is considered a minimum peak if it is preceded (to the left) and followed (to the right) by a higher value. In the analysis of the forces accomplished in this paper, a novel way of detecting peaks was defined. The criterion for detecting maxima and minima used a specified parameter $\alpha$ and was defined as follows:

$$
\begin{gathered}
\text { Maxima }=\{x \mid x>\operatorname{Max} \times \alpha\} \ldots \ldots \ldots \ldots \\
\text { Minima }= \begin{cases}x<\operatorname{Min} \times(1+\alpha) & \text { if } \operatorname{Min}>0 \\
x<\operatorname{Min} \times \alpha & \text { if } \operatorname{Min}<0\end{cases}
\end{gathered}
$$

where Max and Min are the maximum and minimum values of each of the forces for each test and $\alpha$ is a constant value satisfying $0<\alpha<1$. In this case, $\alpha=0.8$ was used. In Table 2, the 20 extracted features for $F_{x}$ are shown (the same was applied to the rest of the forces). Firstly, seven statistical parameters were obtained from the raw signals (all are listed in the table with the exception of cardinality). Afterwards, the maximum and minimum peaks of the signals were identified as described above. The same statistical parameters were obtained from the peaks with the exception of the slope, while in this case the number of peaks per signal, cardinality, was taken into account.

Table 2. Summary of the extracted features for the force $F_{x}$

\begin{tabular}{|c|c|c|c|}
\hline $\begin{array}{c}\text { Statistical } \\
\text { parameters }\end{array}$ & $F_{x}$ & $\begin{array}{c}\text { Maximum } \\
\text { peaks }\end{array}$ & $\begin{array}{c}\text { Minimum } \\
\text { peaks }\end{array}$ \\
\hline Mean & 0 & 7 & 8 \\
\hline Minimum & 1 & 9 & 10 \\
\hline Maximum & 2 & 11 & 12 \\
\hline Variance & 3 & 13 & 14 \\
\hline Median & 4 & 15 & 16 \\
\hline Slope & 5 & - & - \\
\hline Kurtosis & 6 & 17 & 18 \\
\hline Cardinality & - & 19 & 20 \\
\hline
\end{tabular}

\subsection{Applied regression methods}

Various machinelearning algorithms were considered in this research. Some of them belong to the class of ensemble learning techniques and are generally used for both regression and classification. In every case, the regressors used the features described in Table 2. The following regressors were selected due to their extensive applications in other areas: linear regression, decision trees, random forests, $k$-nearest neighbours, adaptive boosting, bootstrap aggregating and 
gradient boosting; however, these algorithms were also applied to a reduced set of initial features by performing feature importance.

Linear regression is one of the most common methods in statistics that linearly relates a dependent variable with one or more independent variables ${ }^{[24]}$.

The $k$-nearest neighbours algorithm is a non-parametric method used for both classification and regression. It is built on the idea of classifying the input data based on a fixed number $k$ of its closest neighbours. The $k$-nearest neighbours regressor estimates the target variable as the average of the values of its $k$-nearest neighbours ${ }^{[2]}$. The most common distance function is the Euclidean distance.

A decision tree is based on a hierarchical decision scheme with a tree-like structure. It is composed of a root node (containing all data), a set of internal nodes (splits) and a set of terminal nodes (leaves). In this approach, features of the data are predictor variables and the class to be predicted is called the target variable. When the target variable is discrete, it is referred to as a decision tree classification, whereas decision tree regression has a continuous target variable ${ }^{[32]}$. The population is split into homogeneous sets or subpopulations, based on the most significant differentiator input variables. These methods present certain advantages, such as the ability to learn non-linear relationships between variables and identify the most influential variables. However, they are less accurate for regression problems than for classification problems. In this investigation, a reduction in variance was used as a splitting criterion.

The random forest regressor is an ensemble learning approach that combines decision trees with the notion of an ensemble. It belongs to the bagging family, which combines the results of multiple classifiers modelled on different subsamples in order to reduce the variance of the predictions. The input data are entered into the system and run down all the trees and the outcome is the weighted average of all of the terminal nodes that have been reached ${ }^{[6]}$. The random forest method can handle large datasets and identify the most significant variables by computing the importance of the variable for the regression.

The bootstrap aggregating regressor is another ensemble learning approach, which also belongs to the bagging family and was considered in this investigation. This algorithm also reduces the error of a learning algorithm, such as a decision tree. In bootstrap aggregating, the learning algorithm is applied to each bootstrap sample of given data ${ }^{[20]}$. The main difference from the random forest method is that this algorithm considers all features for splitting a node. In contrast, the random forest method selects only a subset of all the features at random and the best differentiator input (from the subset) is used to split each node in a tree.

Boosting is a general method for improving the accuracy of a learning algorithm by converting a weak learner into a strong learner. The adaptive boosting regressor is a machine learning boosting technique that uses a weighted sum to obtain the final output, modifying in its favour testing points that present higher errors ${ }^{[22]}$. It starts by predicting the original data, giving equal weight to each observation. If the error of the prediction of a sample is high using the first learner, then a higher weight is given to the observation. The iterative process continues, adding learner(s) until a limit (number of models or accuracy) is reached. In this investigation, the base learning algorithm employed was the decision tree.

Finally, the gradient-boosting regressor, an ensemble learning algorithm that belongs to the family of boosting, was considered. This also starts by giving the same weight to all observations. It uses a weak regressor and the population distribution is updated at each stage. This approach allows differentiable loss functions that map samples to a real number that represents the cost. The loss function is minimised for each new model using the gradient descent method ${ }^{[10]}$.

\subsection{Model validation}

To define the training and testing sets, the leave-one-out crossvalidation technique was used. In $k$-fold cross-validation, the dataset $D$ is randomly split into $k$ subsets (the folds) $D_{1}, D_{2}, \ldots, D_{k}$ of approximately equal size. The sets are used for training and testing $k$ times; at each time $t \in\{1,2, \ldots, k\}$ the regressor is trained on $D \backslash D_{t}$ and tested on $D_{t}$. The leave-one-out corresponds to $n$-fold cross-validation when $k=n$, with $n$ being the number of observations ${ }^{[14]}$. This decision was taken due to the small number of test cases for each state and lubrication, hence the training set could contain $n-1$ samples at each iteration.

The root mean squared error (RMSE) was used to calculate the error, the square root of the mean square error (MSE). The MSE is a measure of how close a fitted value is to the data point. It measures the average of the squares of the errors (the differences between the observed and fitted values $)^{[24]}$. If $\hat{\mathbf{y}}=\left(\hat{y}_{1}, \ldots, \hat{y}_{n}\right)$ is a vector of $n$ predictions and $\mathbf{y}=\left(y_{1}, \ldots, y_{n}\right)$ is the vector of observed values, then the RMSE is calculated in the following way:

$$
R M S E=\sqrt{\frac{1}{n} \sum_{i=1}^{n}\left(\hat{y}_{i}-y_{i}\right)^{2}} .
$$

\section{Results and discussion}

The results of the proposed approach are presented in two parts, the first part involving the LGA microstructure and the second part involving the SGA microstructure. In both states, a comparison is made between the predictions obtained for normal and HPC lubrications. Also, there is a discussion about which regression method is the best for future evaluations of tool wear in the face turning of Inconel 718.

\subsection{Large grain aged}

The aforementioned regression methods were applied to each available test of a specific state and lubrication. The data for the regression problems were set up by splitting the data into two matrices: the input matrix and the target matrix. The input matrix was made up of 63 features (columns) and 12 test cases (rows). The target matrix contained the maximum flank wear measurements.

The RMSE measure allowed for determination of which of the applied methods produced the best prediction. The RMSE values computed from the outputs of these methods are shown in Table 3. The numbers in bold correspond to the lowest RMSE value obtained for each state and lubrication.

One first observation from the analysis of Table 3 is that the errors are quite large for most states and regressors. Before reducing the feature subspace, the algorithm for which the lowest error was generally achieved was the gradient-boosting regressor. The fact that none of the regressors provided consistent and good results illustrates the difficulty of the regression task.

In order to have a better understanding of the quality of the predictions, the real values versus the predicted values for the gradient-boosting regressor are represented in Figure 3(a), as it produced the lowest RMSE value for LGA, normal lubrication. A test is a repetition of the process of turning, which involves six passes for a specific lubrication and state.

An analysis of Table 3 reveals that the RMSE values are much lower for HPC than those obtained for normal lubrication. The lowest RMSE value was obtained using the decision tree regressor. In Figure 3(b), the predicted versus the real values of this regressor are plotted. If this is compared with the Figure that corresponds 
to normal lubrication, Figure 3(a), then the accuracy achieved for HPC is considerably better. The predictions for the first two passes for test 2 are remarkably accurate.

Table 3. RMSE $(\mu \mathrm{m})$ in the prediction of flank wear for each of the regressors and reduced feature subspace learnt from $\mathrm{RF}$, Inconel 718

\begin{tabular}{|c|c|c|c|c|}
\hline Regressor & LGA-N & LGA-H & SGA-N & SGA-H \\
\hline Random forests & 31.08 & 10.47 & 47.79 & 11.90 \\
\hline Linear regression & 68.87 & 7.76 & 238.22 & 16.70 \\
\hline Ada boost & 34.83 & 12 & 56.63 & 11.87 \\
\hline kneighbours & 31.53 & 11.02 & 44.17 & 12.09 \\
\hline Bagging & 59.80 & 16.37 & 72.38 & 16.58 \\
\hline Decision tree & 43.99 & 6.29 & 55.60 & 17.54 \\
\hline Gradient boosting & 29.58 & 8.62 & $\mathbf{3 6 . 5 4}$ & $\mathbf{9 . 8 9}$ \\
\hline Reduced feature subspace & $\mathbf{2 8 . 0 1}$ & $\mathbf{4 . 5 6}$ & 50.14 & 20.10 \\
\hline
\end{tabular}

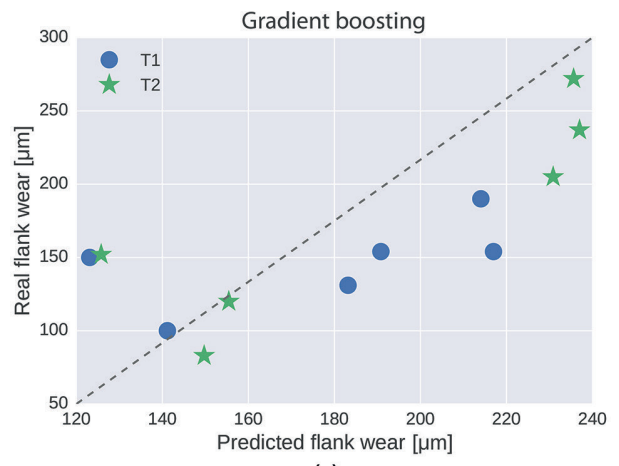

(a)

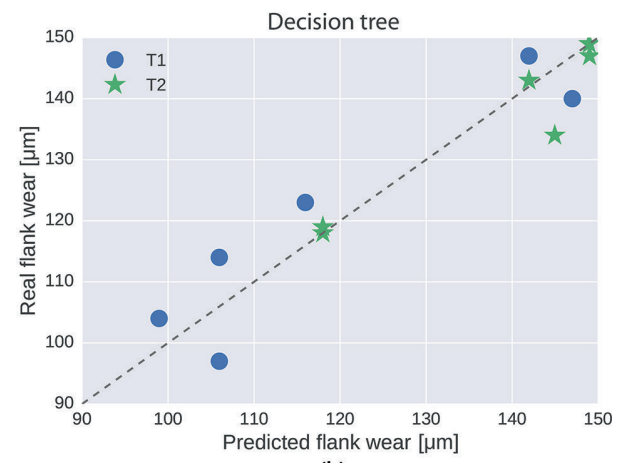

(b)

Figure 3. Real and predicted values using the gradient-boosting regressor and the decision tree regressor for Inconel 718, LGA: (a) normal lubrication; and (b) high-pressure lubrication

The relatively modest results for the regressor approaches in the case of normal lubrication might be due to the highly complex nature of the prediction task, the small amount of data available or the existence of redundant or uninformative features. The question of whether a more rigorous selection of features could further improve the accuracy of the predictions was investigated. For this purpose, feature importance was computed by applying a random forest regressor. Feature selection was carried out using a threshold value $\lambda$. Those features that presented an importance value lower than $\lambda$ were removed.

Firstly, the data from the first test (including the six observations) were utilised to learn the parameters of the random forest regressor and compute feature importance, then all of the considered regressors were applied and validated with the second test using the reduced feature subspace. As leave-one-out cross-validation was used to define the training and testing tests, six estimates were obtained of the feature importance (one vector with the feature importance estimates for each of the six leave-one-out model learning steps), which provided their importance. For each feature, the predicted feature importance was the mean of the six estimates associated with each model learnt.

All regression methods previously considered were applied using a reduced set of selected features with a threshold value of $\lambda=0.02$. In Tables 4 and 5 , the features selected from the feature importance values estimated by the random forest regressor are shown. The RMSE values obtained from the reduced feature subspace are lower than those obtained using the original feature space (Table 3). An analysis of Table 4 reveals that, in the case of normal lubrication, about half of the features correspond to the force $F_{x}$, though the two most important features are related to $F_{y}$. There are some statistical parameters that are repeated, such as the variance and the kurtosis. The distance of the peaks from the mean (variance) and the pointedness of the distribution seem to be good predictors of the flank wear.

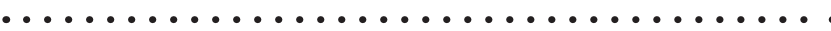

Table 4. Feature importance, LGA, normal

\begin{tabular}{|c|c|c|}
\hline Feature name & Force & Importance \\
\hline Variance of maximum peaks & $F_{y}$ & 0.038597 \\
\hline Variance of minimum peaks & $F_{y}$ & 0.026775 \\
\hline Minimum of maximum peaks & $F_{x}$ & 0.025934 \\
\hline Maximum & $F_{x}$ & 0.025866 \\
\hline Mean & $F_{x}$ & 0.024323 \\
\hline Number of minimum peaks & $F_{y}$ & 0.023912 \\
\hline Median & $F_{x}$ & 0.022984 \\
\hline Mean of maximum peaks & $F_{y}$ & 0.022320 \\
\hline Median & $F_{y}$ & 0.022133 \\
\hline Median of maximum peaks & $F_{y}$ & 0.021130 \\
\hline Slope & $F_{z}$ & 0.021077 \\
\hline Kurtosis of minimum peaks & $F_{x}$ & 0.020521 \\
\hline Mean of maximum peaks & $F_{y}$ & 0.020248 \\
\hline
\end{tabular}

Table 5. Feature importance, LGA, HPC

\begin{tabular}{|c|c|c|}
\hline Feature name & Force & Importance \\
\hline Number of minimum peaks & $F_{x}$ & 0.033352 \\
\hline Number of minimum peaks & $F_{z}$ & 0.028051 \\
\hline Minimum of minimum peaks & $F_{x}$ & 0.027090 \\
\hline Median of minimum peaks & $F_{z}$ & 0.026547 \\
\hline Kurtosis of minimum peaks & $F_{y}$ & 0.024355 \\
\hline Variance of maximum peaks & $F_{z}$ & 0.020804 \\
\hline Minimum of maximum peaks & $F_{x}$ & 0.022586 \\
\hline Median of maximum peaks & $F_{x}$ & 0.021630 \\
\hline Minimum & $F_{x}$ & 0.020886 \\
\hline Maximum of minimum peaks & $F_{z}$ & 0.020804 \\
\hline Median & $F_{z}$ & 0.0206 \\
\hline
\end{tabular}

In the case of HPC, Table 5, the majority of the features correspond to statistical parameters of the distribution of the peaks and the cardinality of the peaks seems to play a relevant role in the regression. Seven of the eleven features are parameters related to minimum peaks. The kurtosis computed for the minimum peaks of 
$F_{y}$ and the minimum of the maximum peaks of $F_{x}$ are the features repeated in both reduced feature subspaces.

\subsection{Small grain aged}

In the case of the SGA state, two tests were available for normal lubrication (12 samples) and three tests for HPC (18 samples), as each test was composed at six passes. Figure 4(a) shows that for the first test the predicted values are higher than the real values in general, except for the first and last passes. For the case of SGA and normal lubrication, the flank wear becomes critical earlier than in the LGA state. This phenomenon can be observed in Figure 5.

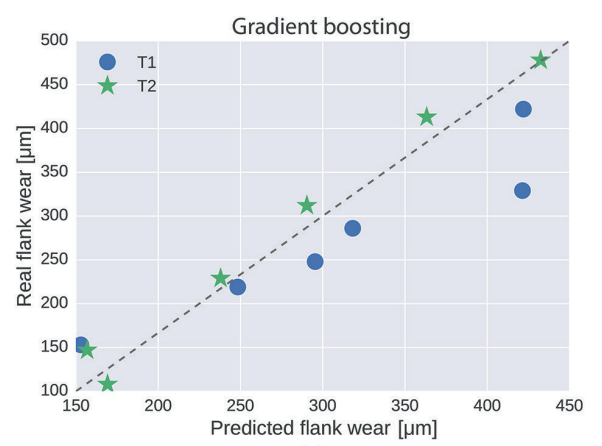

(a)

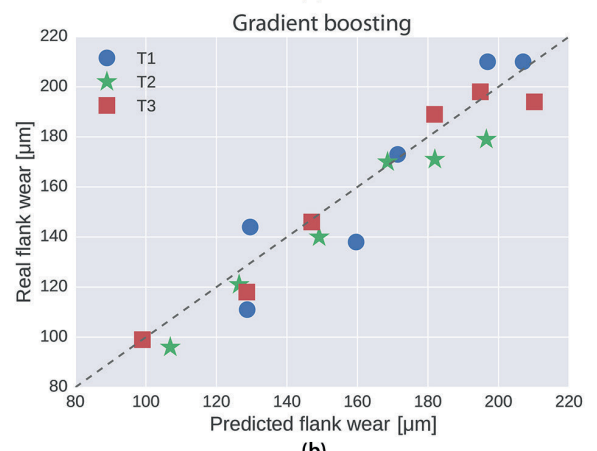

(b)

Figure 4. Real and predicted values using the gradient-boosting regressor for Inconel 718, SGA: (a) normal lubrication; and (b) high-pressure lubrication

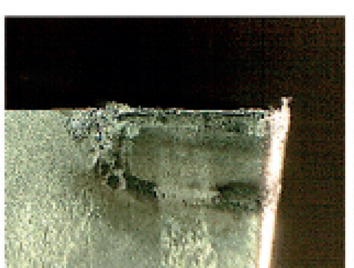

(a)

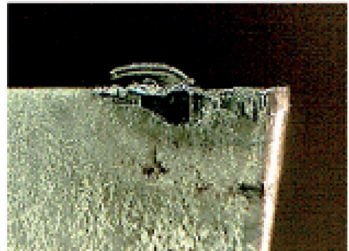

(b)
Figure 5. Turning tool used in the experiments: (a) large grain aged; and (b) small grain aged

Analysis of Table 3 shows that in the case of SGA and HPC lubrication, the RMSE obtained from the method is lower than that obtained for data corresponding to normal lubrication. Figure 4(b) shows real and predicted values for the gradient-boosting regressor with the original feature space and HPC lubrication. It can be observed that the prediction for the sixth pass is higher than the real value for tests 2 and 3 . There are some remarkable cases, such as pass 4 from test 2 , and passes 1,3 and 5 from test 3 , in which the prediction achieves the exact value.

As before, the feature importance was computed using the random forest regressor for the first test and the regressors were trained and tested with the reduced feature subspace for the second.
Tables 6 and 7 show the most important features produced by the method, using $\lambda=0.02$ as a threshold value.

In the case of normal lubrication, the three most important features correspond to the cardinality of the minimum peaks. Similar to the LGA state, there are more features related to the minimum peaks of the cutting forces than to the maximum peaks. In the case of HPC, most of the selected features correspond to the force $F_{y}$. In this case, there are more features related to the maximum peaks than to the minimum peaks. The performance in terms of RMSE is no better when using the reduced feature subspace.

Table 6. Feature importance, SGA, normal

\begin{tabular}{|r|c|c|}
\hline Feature name & Force & Importance \\
\hline Number of minimum peaks & $F_{z}$ & 0.039885 \\
\hline Number of minimum peaks & $F_{x}$ & 0.030493 \\
\hline Number of minimum peaks & $F_{y}$ & 0.027503 \\
\hline Mean & $F_{x}$ & 0.026255 \\
\hline Median & $F_{x}$ & 0.025868 \\
\hline Maximum of minimum peaks & $F_{x}$ & 0.023882 \\
\hline Number of minimum peaks & $F_{x}$ & 0.023688 \\
\hline Slope & $F_{y}$ & 0.023180 \\
\hline Variance of minimum peaks & $F_{z}$ & 0.022635 \\
\hline Kurtosis of minimum peaks & $F_{x}$ & 0.022365 \\
\hline Variance & $F_{y}$ & 0.022349 \\
\hline Minimum of minimum peaks & $F_{y}$ & 0.021864 \\
\hline Minimum of maximum peaks & $F_{x}$ & 0.020581 \\
\hline Minimum of minimum peaks & $F_{x}$ & 0.020427 \\
\hline Variance of maximum peaks & $F_{x}$ & 0.020079 \\
\hline
\end{tabular}

Table 7. Feature importance, LGA, HPC

\begin{tabular}{|r|c|c|}
\hline Feature name & Force & Importance \\
\hline Maximum of minimum peaks & $F_{y}$ & 0.041207 \\
\hline Number of maximum peaks & $F_{y}$ & 0.037089 \\
\hline Kurtosis & $F_{y}$ & 0.035564 \\
\hline Variance of maximum peaks & $F_{y}$ & 0.030151 \\
\hline Number of minimum peaks & $F_{y}$ & 0.029909 \\
\hline Number of maximum peaks & $F_{z}$ & 0.027606 \\
\hline Median of maximum peaks & $F_{z}$ & 0.026286 \\
\hline Number of maximum peaks & $F_{x}$ & 0.026138 \\
\hline Variance & $F_{x}$ & 0.025207 \\
\hline
\end{tabular}

There are several factors that influence tool wear, including cutting tool geometry, cutting conditions, cutting tool material and the workpiece. As the workpiece presents different states in the experiments, differences are expected in the tool wear among both states and lubrications. Regarding the maximum flank wear, the values were considerably higher in both states when using normal lubrication than when using HPC. In the case of HPC, the maximum values were reached in the sixth pass and the pattern was similar for both tests, though for normal lubrication the flank wear for the sixth pass was higher in the second test. It can be concluded that the tests involving HPC lubrication were more similar to one another than those involving normal lubrication. As a result, the quality of the predictions was better in terms of RMSE. There are two features 
that appeared in both reduced subspaces: the minimum of the maximum peaks of $F_{x}$ and the kurtosis of the minimum peaks of $F_{y}$.

Subsequently, predictions for the SGA state for both normal and HPC lubrication were carried out. As for the LGA state when HPC lubrication was used, the prediction of the flank wear was better in terms of RMSE. In the case of the SGA state, both reduced subspaces (normal and HPC) had two features in common: the number of minimum peaks of $F_{y}$ and the number of maximum peaks of $F_{x}$. Generally, the gradient-boosting regressor excelled in quality compared with the other applied regression methods and the reduced feature subspace only produced a gain in the LGA state. Accordingly, in future evaluations the gradient-boosting regressor should be employed.

\section{Conclusions}

This investigation has presented a predictive tool wear model, which, among other things, has served to examine the best (in terms of RMSE) regression method to use in future evaluations. In addition, a novel approach has been proposed that includes feature extraction and machine learning-based feature selection steps and a variety of machine learning techniques to predict flank wear. To begin with, a novel feature extraction of the cutting force signals was performed. This focused on peak detection, as peaks are descriptors of significant events and changes in time series. The improvement achieved in tool wear accuracy, for the LGA state, employing the feature importance tool of the random forest regressor, indicates that the features suitably characterise the turning process. Those features that presented higher importance or were repeated in different models should be studied in more detail. The majority of the selected important features corresponded to $F_{x}$, though there were also several features related to $F_{y}$. Only seven features related to $F_{z}$ appeared. Therefore, there is no doubt of the influence that the radial force $F_{x}$ has on the tool wear.

The regression methods were then applied to the data. It should be noted that it was difficult to develop a predictive model for wear prediction, including the effect of cutting forces, due to the nonlinear behaviour of the wear mechanism, which was influenced by the large number of factors that have an impact on wear. Other elements of added difficulty were the issues concerning the small amount of data available and the noise in the force signals. In spite of the associated difficulty of the process, which affected the prediction task, some significant differences were found among the machine learning approaches used. The ensemble learning techniques, bagging and boosting combine base learners, such as decision trees, or convert weak learners into strong learners. Thus, the ensemble learning methods performed better in terms of RMSE than linear or decision tree regressors. In almost every case, the best method was the gradient-boosting regressor and it was concluded that this is the best method in terms of RMSE to apply in future investigations.

Finally, this investigation contributes to the knowledge of how the characteristics of the material influence the amount of wear. Regarding this, remarkably better results were obtained for HPC lubrication than for normal lubrication in both of the states considered. This phenomenon can be attributed to the fact that for HPC lubrication the different tests were very similar to one another, exhibiting the same pattern as well as reaching the same maximum value in the sixth pass. However, more experiments should be conducted in order to assure repeatability for HPC lubrication. This research work aims to be a first step towards the optimisation of the process. The results obtained can be used in a decision-making system that lengthens the tool life and benefits both productivity and cost.

This study has revealed the necessity of expanding research on
HPC lubrication, as the best results were achieved for this method. This would be a necessary step in the creation of more efficient machining processes. The research presented can be improved with the inclusion of other input variables, such as temperature, and characterising the different states with their respective grain sizes and microhardnesses. Also, it would be interesting to try other models, such as neural networks or regression methods that are based on Gaussian kernels, to investigate their performance for this problem.

\section{Acknowledgements}

The work was performed as a part of the HIMMOVAL project (grant agreement number: 620134) within the Clean Sky programme, which relates to the SAGE2 project oriented to geared open rotor development, enabling the delivery of the demonstrator part. The work of Roberto Santana has been funded by the IT-609-13 programme (Basque Government) and TIN2016-78365-R (Spanish Ministry of Economy, Industry and Competitiveness).

\section{References}

1. Y Arisoy and T Ozel, 'Machine learning-based predictive modelling of machining-induced microhardness and grain size in Ti6Al4V alloy', Materials and Manufacturing Processes, Vol 30, No 4, pp 425-433, 2015.

2. V Astakhov, 'Basic definitions and cutting tool geometry, singlepoint cutting tools', In: Geometry of Single-Point Turning Tools and Drills, pp 55-126, Springer, London, 2010.

3. A Attanasio, E Ceretti and C Giardini, 'Analytical models for tool wear prediction during AISI 1045 turning operations', Procedia CIRP, Vol 8, pp 218-223, 2013.

4. A Siddhpura and R Paurobally, 'A review of flank wear prediction methods for tool condition monitoring in a turning process', International Journal of Advanced Manufacturing Technology, Vol 65, No 1-4, pp 371-393, 2013.

5. M Gerdes, D Galar and D Scholz, 'Genetic algorithms and decision trees for condition monitoring and prognosis of A320 aircraft air conditioning, Insight: Non-Destructive Testing and Condition Monitoring, Vol 59, No 8, pp 424-433, 2017.

6. L Breiman, 'Random forests', Machine Learning, Vol 45, No 1, pp 5-32, 2001.

7. S Choudhury and K Kishore, 'Tool wear measurements in turning using force ratio', International Journal of Machine Tools and Manufacture, Vol 40, No 6, pp 899-909, 2000.

8. O Colak, 'Investigation on machining performance of Inconel 718 under high-pressure cooling conditions', Journal of Mechanical Engineering, Vol 58, No 11, pp 683-690, 2012.

9. J Davim, Machining: Fundamentals and Recent Advantages, Springer, 2008.

10. J Friedman, 'Stochastic gradient boosting', Computational Statistics \& Data Analysis, Vol 38, No 4, pp 367-378, 2002.

11. N Ghosh, Y B Ravi, A Patra, S Mukhopadhyay, S Paul, A R Mohanty and A B Chattopadhyay, 'Estimation of tool wear during $\mathrm{CNC}$ milling using neural network-based sensor fusion', Mechanical Systems and Signal Processing, Vol 21, No 1, pp 466-479, 2007.

12. C Hu, G Jain, P Zhang, C Schmidt, P Gomadam and T Gorka, 'Data-driven method based on particle swarm optimisation and $k$-nearest neighbour regression for estimating capacity of lithium-ion battery', Applied Energy, Vol 129, pp 49-55, 2014.

13. B Kilundu, X Dehombreaux and X Chiementin, 'Tool wear monitoring by machine learning techniques and singular spectrum analysis', Mechanical Systems and Signal Processing, Vol 25, No 1, pp 400-415, 2011.

14. R Kohavi, 'A study of cross-validation and bootstrap for accuracy estimation and model selection', Proceedings of the 
14th International Joint Conference on Artificial Intelligence, Montreal, Québec, Canada, pp 1137-1143, 20-25 August 1995.

15. A Kusiak, 'Feature transformation methods in data mining', IEEE Transactions on Electronics Packaging Manufacturing, Vol 24, No 3, pp 214-221, 2001.

16. M Lofti, M Jahanbakhsh and A Farid, 'Wear estimation of ceramic and coated carbide tools in turning of Inconel 625: 3D FE analysis', Tribology International, Vol 99, pp 107-116, 2016.

17. P Mahadeo and U Shanker, Modelling of Metal Forming and Machining Processes: By Finite Element and Soft Computing Methods, Springer, 2008.

18. S Olovsjö and L Nyborg, 'Influence of microstructure on wear behaviour of uncoated WC tools in turning of Alloy 718 and Waspaloy', Wear, Vol 282, pp 12-21, 2012.

19. S Olovsjö, A Wretland and G Sjöberg, 'The effect of grain size and hardness of wrought Alloy 718 on the wear of cemented carbide tools', Wear, Vol 268, No 9, pp 1045-1052, 2010.

20. R Pino-Mejas, M Jimenez-Gamero, M C Cubiles de la Vega and A Pascual-Acosta, 'Reduced bootstrap aggregating of learning algorithms', Pattern Recognition Letters, Vol 29, No 3, pp 265-271, 2008.

21. J J Rodríguez, G Quintana, A Bustillo and J Ciurana, 'A decisionmaking tool based on decision trees for roughness prediction in face milling', International Journal of Computer Integrated Manufacturing, Vol 30, No 9, pp 943-957, 2017.

22. R Schapire, The Boosting Approach to Machine Learning: An Overview, Springer, 2003.

23. F Scholkmann, J Boss and M Wolf, 'An efficient algorithm for automatic peak detection in noisy periodic and quasi-periodic signals', Algorithms, Vol 5, No 4, pp 588-603, 2012.

24. G A Seber and A J Lee, Linear Regression Analysis, Wiley, 2003.

25. D Shi and N Gindy, 'Tool wear predictive model based on leastsquares support vector machines', Mechanical Systems and
Signal Processing, Vol 21, No 4, pp 1799-1814, 1997.

26. S Sikdar and M Chen, 'Relationship between tool flank wear area and component forces in single-point turning, Journal of Materials Processing Technology, Vol 128, No 1, pp 210-215, 2002.

27. R Slavkovic, S Arsovski and A Jovicic, 'A study of wear rate estimation of casting parts by support vector machine', Proceedings of the 16th International Research/Expert Conference, Dubai, United Arab Emirates, pp 619-622, 10-12 September 2012.

28. A Suárez, L N López de Lacalle, R Polvorosa and F Veiga, 'Effects of high-pressure cooling on the wear patterns on turning inserts used on Alloy IN718', Materials and Manufacturing Processes, Vol 32, No 6, pp 678-686, 2017.

29. A Suárez, F Veiga, L N López de Lacalle, R Polvorosa, S Lutze and A Wretland, 'Effects of ultrasonics-assisted face milling on surface integrity and fatigue life of Ni-Alloy 718, Journal of Materials Engineering and Performance, Vol 25, No 11, pp 5076-5086, 2016.

30. N Vaxevanidis, J Kechagias, N Fountas and D Manolakos, 'Three component cutting force system modelling and optimisation in turning of AISI D6 tool steel using design of experiments and neural networks', Proceedings of the World Congress on Engineering, Vol 1, London, UK, pp 629-633, 3-5 July 2013.

31. J Wang, P Wang and R Gao, Enhanced particle filter for tool wear prediction', Journal of Manufacturing Systems, Vol 36, pp 35-45, 2015.

32. $\mathrm{MXu}, \mathrm{P}$ Watanachaturaporn, $\mathrm{P}$ Varshney and $\mathrm{M}$ Arora, 'Decision tree regression for soft classification of remote sensing data, Remote Sensing of Environment, Vol 97, No 3, pp 322-336, 2005.

33. Y Yen, J Söhner, B Lilly and T Altan, 'Estimation of tool wear in orthogonal cutting using the finite element analysis', Journal of Materials Processing Technology, Vol 146, pp 82-91, 2004.

34. D Zhu, XZhang and H Ding, 'Tool wear characteristics in machining of nickel-based superalloys', International Journal of Machine Tools and Manufacture, Vol 64, pp 60-77, 2013. 